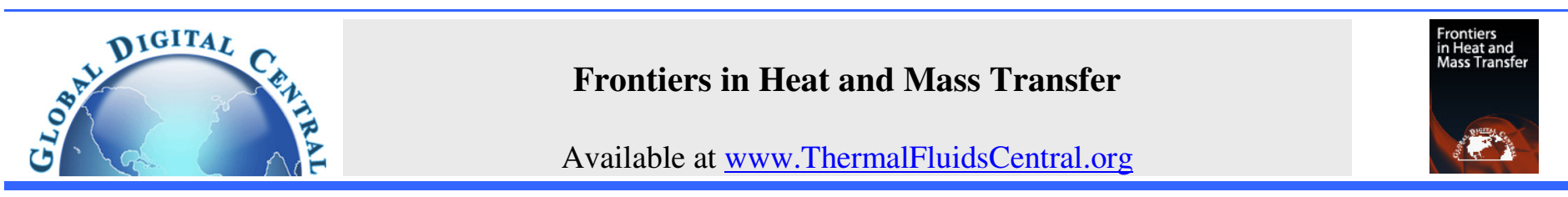

\title{
COMBINED INFLUENCE OF HALL CURRENTS AND JOULE HEATING ON HEMODYNAMIC PERISTALTIC FLOW WITH POROUS MEDIUM THROUGH A VERTICAL TAPERED ASYMMETRIC CHANNEL WITH RADIATION
}

\author{
S. Ravi Kumar*, S. K. Abzal \\ Department of Mathematic, NBKR Institute of Science and Technology, Vidyanagar, SPSR Nellore, 524413, Andhra Pradesh, India.
}

\begin{abstract}
The aim of the present attempt is hall currents and joule heating on peristaltic blood flow in porous medium through a vertical tapered asymmetric channel under the influence of radiation. The Mathematical modeling is investigated by utilizing long wavelength and low Reynolds number assumptions. The indicates an appreciable increase in the axial velocity distribution with increase in hall current parameter and porosity parameter whereas the result in axial velocity distribution diminished by increase in magnetic field parameter. The result in pressure gradient reduces by rise in hall current parameter, porosity parameter and volumetric flow rate. The temperature of the fluid increases by increase in $\mathrm{M}, \mathrm{N}, \mathrm{Pr}$ and $\mathrm{Br}$ and decreases by increase in $\mathrm{m}$ and Da.
\end{abstract}

Keywords: Hall current, porosity parameter, joule heating, radiation and tapered channel

\section{INTRODUCTION}

Past five decades researchers have enormously paid attention to the peristaltic pumping of Newtonian and non-Newtonian fluids. In particular, the discussion of peristaltic fluid flow has created lots of curiosity and hence, good literature is currently available on the subject. A thorough understanding of peristalsis is of great interest, due to its natural property of the numerous biological systems taking sleek muscle tubes which transport biofluids through its propulsive movements. It is found within the movement of food bolus through oesophagus, transport of body waste from urinary organ to the bladder through the channel, the movement of spermatozoa within the ducts afferents of the male reproductive tract, circulation of blood in the small blood vessels, the movement of chyme in the gastrointestinal tract, intra-uterine fluid motion, movements of ovum in the female fallopian tube are some samples of peristaltic fluid flow. In addition, peristaltic pumping occurs in many practical applications involving biomechanical systems. Several attempts had been made to know and understand peristaltic action in different situations. Since the first attempt of Latham (1966) some interesting investigations in this direction have been given in (Fung and Yih, 1968; Shapiro et al., 1969; Jaffrin and Shapiro, 1971; Srivastava and Srivastava, 1984; Agarwal and Anwaruddin, 1984; Shehawey and Husseny, 2002; Siddiqui et al., 2004; El Naby et al., 2006; Mekheimer, 2004; Mishra and Ramachandra Rao, 2003; Haroun, 2007; Vajravelu et al., 2011).

The Hall effect is important when the Hall parameter which is the ratio between the electron-cyclotron frequency and the electron-atomcollision frequency is high; this can occur if the collision frequency is low or when the magnetic field is high. This is a current trend in MHD because of its important influence of the electromagnetic force. Hence, it is important to study Hall effects and heat transfer effects on the flow to be able to determine the efficiency of some devices such as power generators and heat exchangers. Attia (2004) had examined unsteady Hartmann flow with heat transfer of a viscoelastic fluid taking the Hall effect into account. Asghar et al. (2005) studied the effects of Hall current and heat transfer on flow due to a pull of eccentric rotating disk. Hayat et al. (2007) studied the Hall effects on the peristaltic flow of a Maxwell fluid in a porous medium. Hayat et al. (2016) investigated an influence of Hall current and chemical reaction in mixed convective peristaltic flow of Prandtl fluid. For the benefit of the readers in this direction, you can take these additional papers (Akbar and Nadeem, 2010, Ali et al., 2010; Eldabe et al., 2015, Nowar, 2014, Abd El-Maboud and Mekheimer, 2010, Mekheimer et al., 2010; Gharsseldien et al., 2010; Srinivas and Muthuraj, 2011) into account.

The study of the flow of fluids through porous media has the greatest significance for the last one and half centuries because of their occurrence in Sanitary Engineering and newly emerging fields such as Petroleum Industry, Polymer Industry and its wide applications in Physiology and Bio-Mechanics. The petroleum industries are showing good curiosity in the study of the heat and mass transfer of the flow of oil through porous rocks. In order to study the seepage of water in river beds, one requires the knowledge of the flow of fluids through porous media, which is necessary to tap groundwater from the underground reservoirs to overcome the crisis created by the failure of monsoons in the rain-fed areas. Flow through porous media is also useful to the paper industry because, in the papermaking process, felts are used to carry the paper sheet through its many stages of formation and drying.

In general heat transfer, plays a vital role in MHD flows. Heat transfer is that the transition of thermal energy from a section of upper temperature to a locality of lower temperature. Vajravelu et al. (2007) gave a numerical model for peristaltic transportation and heat transfer in a perpendicular porous annulus. In another try, Nadeem et al. (2009) mentioned on an impact of heat transfer on peristalsis with

* Corresponding author. Email: drsravikumar1979@gmail.com 
variable viscosity. Shehzad et al. (2014) investigated on hydromagnetic peristaltic transportation of nanofluid with Joule Heating and thermophoresis. The peristaltic flow of fourth-grade fluid with Dufour and Sore effects was studied by Mustafa et al. (2014). Recently, Ravi Kumar (2016) investigated on hydromagnetic peristaltic transportation through a perpendicular tapered uneven channel with radiation. Some pertinent studies on this topic will be found from the list of Refs. Such as Tasawar Hayat et al. (2017), Kothandapani et al. (2015) Abzal et al. (2016) , Jitendra Kumar Singh et al. (2016) and Veeresh et al. (2017).

\section{FORMULATION OF THE PROBLEM}

We consider the MHD peristaltic transport of an incompressible viscous fluid in a two-dimensional uneven inclined perpendicular tapered channel under the influence of porous medium. The joule heating, hall currents and radiation is taken into the account. The left wall of the channel is maintained at temperature $\mathrm{T}_{0}$, whereas the right wall has temperature $T_{1}$. We tend to assume that the fluid is subject to a relentless transverse magnetic field $\mathrm{B}_{0}$. The fluid is induced by sinusoidal wave trains propagating with constant speed $c$ along the channel walls.

The geometry of the wall deformations are drawn by the subsequent expressions

$Y=\overline{H_{2}}=b+m^{I} \bar{X}+d \sin \left[\frac{2 \pi}{\lambda}(\bar{X}-c \bar{t})\right]$

$Y=\overline{H_{1}}=-b-m^{I} \bar{X}-d \sin \left[\frac{2 \pi}{\lambda}(\bar{X}-c \bar{t})+\phi\right]$

Where $b$ is the half-width of the channel, $d$ is the wave amplitude, $c$ is the phase speed of the wave and $m^{\prime}\left(m^{\prime}<<1\right)$ is the non-uniform parameter, $\lambda$ is the wavelength, $t$ is the time and $X$ are the direction of wave propagation. The phase difference $\phi$ varies in the range $0 \leq \phi \leq \pi$, $\phi=0$ corresponds to the symmetric channel with waves out of phase and further $\mathrm{b}, \mathrm{d}$ and $\phi$ satisfy the following conditions for the divergent channel at the inlet $d \cos \left(\frac{\phi}{2}\right) \leq b$. It is assumed that the left wall of the channel is maintained at temperature $\mathrm{T}_{0}$ while the right wall has temperature $\mathrm{T}_{1}$.

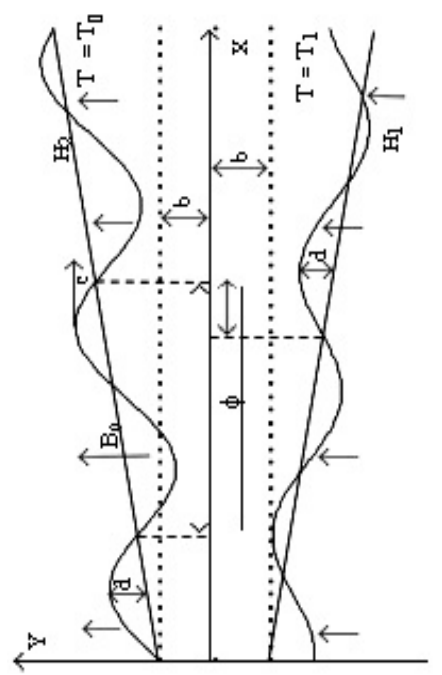

Fig. 1 Schematic diagram of the physical model

The equations governing the motion for the present problem prescribed by

The continuity equation is

$\frac{\partial u}{\partial x}+\frac{\partial v}{\partial y}=0$
The momentum equations are

$$
\begin{aligned}
\rho\left[u \frac{\partial u}{\partial x}+v \frac{\partial u}{\partial y}\right]= & -\frac{\partial p}{\partial x}+u\left[\frac{\partial^{2} u}{\partial x^{2}}+\frac{\partial^{2} u}{\partial y^{2}}\right]+ \\
& {\left[\frac{\sigma B_{0}^{2}}{1+m^{2}}\right](m v-(u+c))-\frac{\mu}{k_{1}}(u+c) } \\
\rho\left[u \frac{\partial v}{\partial x}+v \frac{\partial v}{\partial y}\right]= & -\frac{\partial p}{\partial y}+u\left[\frac{\partial^{2} v}{\partial x^{2}}+\frac{\partial^{2} v}{\partial y^{2}}\right]- \\
& {\left[\frac{\sigma B_{0}^{2}}{1+m^{2}}\right](m(u+c)+v)-\frac{\mu}{k_{1}}(v+c) }
\end{aligned}
$$

The energy equation is

$\rho C_{p}\left[u \frac{\partial}{\partial x}+v \frac{\partial}{\partial y}\right] T=k\left[\frac{\partial^{2}}{\partial x^{2}}+\frac{\partial^{2}}{\partial y^{2}}\right] T+Q_{0}+\sigma B_{0}^{2} u^{2}-\frac{\partial q}{\partial x}$

$\mathrm{u}$ and $\mathrm{v}$ are the velocity components in the corresponding coordinates, $\mathrm{k}_{1}$ is the permeability of the porous medium, $\rho$ is the density of the fluid, $p$ is the fluid pressure, $k$ is the thermal conductivity, $\mu$ is the coefficient of the viscosity, $Q_{0}$ is the constant heat addition/absorption, $C_{p}$ is the specific heat at constant pressure, $\sigma$ is the electrical conductivity, $g$ is the acceleration due to gravity and $\mathrm{T}$ is the temperature of the fluid.

The relative boundary conditions are

$\bar{U}=0, \bar{T}=T_{0}$ at $\bar{Y}=\bar{H}_{1}$

$\bar{U}=0, \bar{T}=T_{1}$ at $\bar{Y}=\bar{H}_{2}$

The radioactive heat flux (Cogley et al (1968)) is given by

$\frac{\partial q}{\partial x}=4 \alpha^{2}\left(T_{0}-T_{1}\right)$

here $\alpha$ is the mean radiation absorption coefficient.

Introducing a wave frame $(\mathrm{x}, \mathrm{y})$ moving with velocity $\mathrm{c}$ away from the fixed frame $(\mathrm{X}, \mathrm{Y})$ by the transformation

$x=X$-ct $, y=Y, u=U-c, v=V$ and $p(x)=P(X, t)$

Introducing the following non-dimensional quantities:

$$
\begin{aligned}
& \bar{x}=\frac{x}{\lambda} \quad \bar{y}=\frac{y}{b} \bar{t}=\frac{c t}{\lambda} \quad \bar{u}=\frac{u}{c} \quad \bar{v}=\frac{v}{c \delta} \quad h_{1}=\frac{H_{1}}{b} \quad h_{2}=\frac{H_{2}}{b} \\
& p=\frac{b^{2} p}{c \lambda \mu} \theta=\frac{T-T_{0}}{T_{1}-T_{0}} \delta=\frac{b}{\lambda} \operatorname{Re}=\frac{\rho c b}{\mu} \quad \eta_{1}=\frac{\rho a_{0}{ }^{3} g}{\lambda \mu c} \\
& M=B_{0} b \sqrt{\frac{\sigma}{\mu}} \operatorname{Pr}=\frac{\mu C_{p}}{k} E_{c}=\frac{c^{2}}{C_{p}\left(T_{1}-T_{0}\right)} N^{2}=\frac{4 \alpha^{2} d_{1}{ }^{2}}{k} \\
& \gamma=\frac{Q_{0} b^{2}}{\mu C_{p}\left(T_{1}-T_{0}\right)} \varepsilon=\frac{d}{b} \eta=\frac{\rho a_{0}{ }^{2} g}{\mu c}
\end{aligned}
$$

where $\epsilon=\frac{d}{b}$ is the non-dimensional amplitude of channel, $\delta=\frac{b}{\lambda}$ is the wave number, $k_{1}=\frac{\lambda m^{I}}{b}$ is the non - uniform parameter, $\operatorname{Re}$ is the Reynolds number, $\mathrm{M}$ is the Hartman number, $K=\frac{k}{b^{2}}$ Permeability parameter, $\operatorname{Pr}$ is the Prandtl number, $E_{c}$ is the Eckert number, $\gamma$ is the heat source/sink parameter, $\mathrm{B}_{\mathrm{r}}\left(=\mathrm{E}_{\mathrm{c}} \mathrm{P}_{\mathrm{r}}\right)$ is the Brinkman number, $\eta$ and $\eta_{1}$ are gravitational parameters and $N^{2}$ is the radiation parameter.

\section{SOLUTION OF THE PROBLEM}

In view of the above transformations (8) and non-dimensional variables (9), equations (3-6) are reduced to the following non-dimensional form after dropping the bars,

$$
\begin{aligned}
\operatorname{Re} \delta\left[u \frac{\partial u}{\partial x}+v \frac{\partial u}{\partial y}\right]= & -\frac{\partial p}{\partial x}+\delta^{2} \frac{\partial^{2} u}{\partial x^{2}}+\frac{\partial^{2} u}{\partial y^{2}}+ \\
& \frac{M^{2}}{1+m^{2}}(m \delta v-(u+1))-\frac{1}{D} u-\frac{1}{D} \\
R e \delta^{3}\left[u \frac{\partial v}{\partial x}+v \frac{\partial v}{\partial y}\right]= & -\frac{\partial p}{\partial y}+\delta^{2}\left(\delta^{2} \frac{\partial^{2} v}{\partial x^{2}}+\frac{\partial^{2} v}{\partial y^{2}}\right)- \\
\operatorname{Re}\left[\delta u \frac{\partial \theta}{\partial x}+v \frac{\partial \theta}{\partial y}\right]= & \frac{1}{P_{r}}\left[\delta^{2} \frac{\partial^{2} \theta}{\partial x^{2}}+\frac{\partial^{2} \theta}{\partial y^{2}}\right]+\beta+M^{2} E_{c} u^{2}+\frac{N^{2} \theta}{P_{r}}
\end{aligned}
$$


Applying long wave length approximation and neglecting the wave number along with low-Reynolds numbers. Equations (10-12) become

$\frac{\partial^{2} u}{\partial y^{2}}-\left(\frac{M^{2}}{1+m^{2}}+\frac{1}{D}\right) u=\frac{\partial p}{\partial x}+\left(\frac{M^{2}}{1+m^{2}}+\frac{1}{D}\right)$

$\frac{\partial p}{\partial y}=0$

$\frac{1}{P_{r}} \frac{\partial^{2} \theta}{\partial y^{2}}+\beta+M^{2} E_{c} u^{2}+\frac{N^{2} \theta}{P_{r}}=0$

The relative boundary conditions in dimensionless form are given by

$\mathrm{u}=-1, \theta=0$ at $y=h_{1}=1-k_{1} x-\varepsilon \sin [2 \pi(x-t)+\phi]$

$\mathrm{u}=-1, \theta=1$ at $y=h_{2}=1+k_{1} x+\varepsilon \sin [2 \pi(x-t)]$

The solutions of velocity and temperature with subject to boundary conditions (16) and (17) are given by

$u=a_{1} \sinh \left[\alpha_{1} y\right]+\cosh \left[\alpha_{1} y\right]+a$

Where

$a_{1}=\left(\frac{(1+a)}{\sinh \left[\alpha_{1} h_{2}\right]}\right)+$

$\left[\frac{\cosh \left[\alpha_{1} h_{2}\right](1+a)}{\left(\sinh \left[\alpha_{1} h_{2}\right]\right)\left(\frac{\cosh \left[\alpha_{1} h_{1}\right]-\cosh \left[\alpha_{1} h_{2}\right]}{\sinh \left[\alpha_{1} h_{1}\right]-\sinh \left[\alpha_{1} h_{2}\right]}\right) \sinh \left[\alpha_{1} h_{1}\right]-\cosh \left[\alpha_{1} h_{1}\right]}\right]$

$a_{2}=\left[\frac{(1+a)}{\left(\frac{\cosh \left[\alpha_{1} h_{1}\right]-\cosh \left[\alpha_{1} h_{2}\right]}{\sinh \left[\alpha_{1} h_{1}\right]-\sinh \left[\alpha_{1} h_{2}\right]}\right) \sinh \left[\alpha_{1} h_{1}\right]-\cosh \left[\alpha_{1} h_{1}\right]}\right]$

$a=-\left[1+\left(\frac{p}{\frac{M^{2}}{1+m^{2}}+\frac{1}{D a}}\right)\right]$, where $p=\frac{\partial p}{\partial x}$

$\theta=a_{9} \cos [N y]+a_{8} \sin [N y]-\frac{\beta P_{r}}{N^{2}}-\left[\frac{M^{2} B_{r} a_{3}}{4 \alpha_{1}^{2}+N^{2}}\right] e^{2 \alpha_{1} y}-$

$\left[\frac{M^{2} B_{r} a_{4}}{4 \alpha_{1}^{2}+N^{2}}\right] e^{-2 \alpha_{1} y}-\left[\frac{M^{2} B_{r} a_{5}}{\alpha_{1}^{2}+N^{2}}\right] e^{\alpha_{1} y}-\left[\frac{M^{2} B_{r} a_{6}}{\alpha_{1}^{2}+N^{2}}\right] e^{-\alpha_{1} y}-\frac{M^{2} B_{r} a_{7}}{N^{2}}$

Where

$a_{3}=\left[\frac{a_{1}^{2}}{4}+\frac{a_{2}^{2}}{4}+\frac{a_{1} a_{2}}{2}\right], a_{4}=\left[\frac{a_{1}^{2}}{4}+\frac{a_{2}^{2}}{4}-\frac{a_{1} a_{2}}{2}\right]$

$a_{5}=\left[a\left(a_{1}+a_{2}\right)\right], a_{6}=\left[a\left(a_{2}-a_{1}\right)\right]$

$a_{7}=\left[-\frac{a_{1}^{2}}{2}+\frac{a_{2}^{2}}{2}+a^{2}\right]$

$a_{8}=\left[\frac{-\cos \left[N h_{1}\right]-a_{10}-a_{11}-a_{12}-a_{13}-a_{14}-a_{15}}{a_{16}}\right]$

$a_{9}=\left[\frac{-a_{8} \sin \left[N h_{1}\right]+\frac{\beta P_{r}}{N^{2}}+\left[\frac{M^{2} B_{r} a_{3}}{4 \alpha_{1}{ }^{2}+N^{2}}\right] e^{2 \alpha_{1} h_{1}}}{\cos \left[N h_{1}\right]}\right]+$

$\left[\frac{\left[\frac{M^{2} B_{r} a_{4}}{4 \alpha_{1}^{2}+N^{2}}\right] e^{-2 \alpha_{1} h_{1}}+\left[\frac{M^{2} B_{r} a_{5}}{\alpha_{1}^{2}+N^{2}}\right] e^{\alpha_{1} h_{1}}+\left[\frac{M^{2} B_{r} a_{6}}{\alpha_{1}^{2}+N^{2}}\right] e^{-\alpha_{1} h_{1}}+\frac{M^{2} B_{r} a_{7}}{N^{2}}}{\cos \left[N h_{1}\right]}\right]$

$a_{10}=\left[\left(\frac{\beta P_{r}}{N^{2}}\right)\left(\cosh \left[N h_{1}\right]-\cosh \left[N h_{2}\right]\right)\right]$

$a_{11}=\left[\left(\frac{M^{2} B_{r} a_{3}}{4 \alpha_{1}^{2}+N^{2}}\right)\left(e^{2 \alpha_{1} h_{2}} \cosh \left[N h_{1}\right]-e^{2 \alpha_{1} h_{2}} \cosh \left[N h_{1}\right]\right)\right]$

$a_{12}=\left[\left(\frac{M^{2} B_{r} a_{4}}{4 \alpha_{1}^{2}+N^{2}}\right)\left(e^{-2 \alpha_{1} h_{2}} \cosh \left[N h_{1}\right]-e^{-2 \alpha_{1} h_{2}} \cosh \left[N h_{1}\right]\right)\right]$

$a_{13}=\left[\left(\frac{M^{2} B_{r} a_{5}}{\alpha_{1}^{2}+N^{2}}\right)\left(e^{\alpha_{1} h_{2}} \cosh \left[N h_{1}\right]-e^{\alpha_{1} h_{2}} \cosh \left[N h_{1}\right]\right)\right]$

$a_{14}=\left[\left(\frac{M^{2} B_{r} a_{6}}{\alpha_{1}^{2}+N^{2}}\right)\left(e^{-\alpha_{1} h_{2}} \cosh \left[N h_{1}\right]-e^{-\alpha_{1} h_{2}} \cosh \left[N h_{1}\right]\right)\right]$

$a_{15}=\left[\left(\frac{M^{2} B_{r} a_{7}}{N^{2}}\right)\left(\cosh \left[N h_{1}\right]-\cosh \left[N h_{1}\right]\right)\right]$

$a_{16}=\left[\sinh \left[N h_{1}\right] \cos \left[N h_{2}\right]-\sinh \left[N h_{2}\right] \cos \left[N h_{1}\right]\right]$
The coefficients of the heat transfer $\mathrm{Zh}_{1}$ and $\mathrm{Zh}_{2}$ at the walls $\mathrm{y}=\mathrm{h}_{1}$ and $\mathrm{y}=\mathrm{h}_{2}$ respectively, are given by

$Z h_{1}=\theta_{y} h_{1 x}$

$Z h_{2}=\theta_{y} h_{2 x}$

The solutions of the coefficient of heat transfer at $y=h_{1}$ and $y=h_{2}$ are given by

$Z h_{1}=\theta_{y} h_{1 x}=$

$\left(-a_{9} N \sin [N y]+a_{8} N \cos [N y]-\left[\frac{2 \alpha_{1} M^{2} B_{r} a_{3}}{4 \alpha_{1}^{2}+N^{2}}\right] e^{2 \alpha_{1} y}+\right.$

$\left.\left[\frac{2 \alpha_{1} M^{2} B_{r} a_{4}}{4 \alpha_{1}^{2}+N^{2}}\right] e^{-2 \alpha_{1} y}-\left[\frac{\alpha_{1} M^{2} B_{r} a_{5}}{\alpha_{1}^{2}+N^{2}}\right] e^{\alpha_{1} y}+\left[\frac{\alpha_{1} M^{2} B_{r} a_{6}}{\alpha_{1}^{2}+N^{2}}\right] e^{-\alpha_{1} y}\right)$

$\left(-2 \pi \cos [2 \pi(x-t)+\emptyset]-k_{1}\right)$

$Z h_{2}=\theta_{y} h_{2 x}=$

$\left(-a_{9} N \sin [N y]+a_{8} N \cos [N y]-\left[\frac{2 \alpha_{1} M^{2} B_{r} a_{3}}{4 \alpha_{1}^{2}+N^{2}}\right] e^{2 \alpha_{1} y}+\right.$

$\left.\left[\frac{2 \alpha_{1} M^{2} B_{r} a_{4}}{4 \alpha_{1}^{2}+N^{2}}\right] e^{-2 \alpha_{1} y}-\left[\frac{\alpha_{1} M^{2} B_{r} a_{5}}{\alpha_{1}^{2}+N^{2}}\right] e^{\alpha_{1} y}+\left[\frac{\alpha_{1} M^{2} B_{r} a_{6}}{\alpha_{1}^{2}+N^{2}}\right] e^{-\alpha_{1} y}\right)$

$\left(2 \pi \varepsilon \cos [2 \pi(x-t)]+k_{1}\right)$

The volumetric flow rate in the wave frame is defined by

$q=\int_{h_{1}}^{h_{2}}\left(a_{1} \sinh \left[\alpha_{1} y\right]+\cosh \left[\alpha_{1} y\right]+a\right) d y$

$\frac{a_{1}}{\alpha_{1}}\left[\cosh \left[\alpha_{1} h_{2}\right]-\cosh \left[\alpha_{1} h_{1}\right]\right]+\frac{a_{2}}{\alpha_{1}}\left[\sinh \left[\alpha_{1} h_{2}\right]-\sinh \left[\alpha_{1} h_{1}\right]\right]+$

$\left[a\left(h_{2}-h_{1}\right)\right]$

The pressure gradient obtained from equation (24) can be expressed as

$\frac{d p}{d x}=\left[\frac{M^{2}}{1+m^{2}}+\frac{1}{D}\right]-\left[\frac{q+B E-D F}{\left(h_{2}-h_{1}\right)+D F-B E}\right]\left[\frac{M^{2}}{1+m^{2}}+\frac{1}{D}\right]$

The instantaneous flux $\mathrm{Q}(\mathrm{x}, \mathrm{t})$ in the laboratory frame is

$Q=\int_{h_{2}}^{h_{1}}(u+1) d y=q-h$

The average volume flow rate over one wave period $(\mathrm{T}=\lambda / \mathrm{c})$ of the peristaltic wave is defined as

$\bar{Q}=\frac{1}{T} \int_{0}^{T} Q d t=q+1+d$

From the equations (25) and (27), the pressure gradient can be expressed as

$\frac{d p}{d x}=\left[\frac{M^{2}}{1+m^{2}}+\frac{1}{D}\right]-\left[\frac{(\bar{Q}-1-d)+B E-D F}{\left(h_{2}-h_{1}\right)+D F-B E}\right]\left[\frac{M^{2}}{1+m^{2}}+\frac{1}{D}\right]$

Where

$$
\begin{aligned}
& B=\left[1+\left[\frac{\cosh \left[\alpha_{1} h_{2}\right]}{\left(\frac{\cosh \left[\alpha_{1} h_{1}\right]-\cosh \left[\alpha_{1} h_{2}\right]}{\sinh \left[\alpha_{1} h_{1}\right]-\sinh \left[\alpha_{1} h_{2}\right]}\right) \sinh \left[\alpha_{1} h_{1}\right]-\cosh \left[\alpha_{1} h_{1}\right]}\right]\right] \\
& D=\left[\frac{1}{\left.\left(\frac{\cosh \left[\alpha_{1} h_{1}\right]-\cosh \left[\alpha_{1} h_{2}\right]}{\sinh \left[\alpha_{1} h_{1}\right]-\sinh \left[\alpha_{1} h_{2}\right]}\right) \sinh \left[\alpha_{1} h_{1}\right]-\cosh \left[\alpha_{1} h_{1}\right]\right]}\right] \\
& E=\left[\frac{\cosh \left[\alpha_{1} h_{2}\right]-\cosh \left[\alpha_{1} h_{1}\right]}{\alpha_{1} \sinh \left[\alpha_{1} h_{2}\right]}\right] \quad F=\left[\frac{\sinh \left[\alpha_{1} h_{2}\right]-\sinh \left[\alpha_{1} h_{1}\right]}{\alpha_{1}}\right]
\end{aligned}
$$

\section{DISCUSSION OF THE PROBLEM}

The objective of this research is to study hall currents and joule heating on peristaltic blood flow in the porous medium through a vertical tapered asymmetric channel under influence of radiation. In order to find out numerical solutions, MATHEMATICA software is used.

Fig. 2 indicate the behaviour of axial velocity with y for different values of hall current parameter $\mathrm{m}$ with fixed $\mathrm{Da}=0.1, \mathrm{M}=2, \mathrm{k}_{1}=0.1$, $\mathrm{dp} / \mathrm{dx}=-1, \mathrm{t}=0.4, \varnothing=\pi / 6, \mathrm{x}=0.6, \varepsilon=0.2$. It is clear from the figure that the velocity gradually enhances by increase in Hall current parameter $\mathrm{m}$. 
The impact of porosity parameter $\mathrm{Da}$ on axial velocity distribution is shown in figure 3 . We perceive from this graph that the axial velocity distribution gradually increases by increase in porosity parameter $\mathrm{Da}$ (Da $=0.1,0.2,0.3)$. Fig.4 depicts the axial velocity distribution (u) with dissimilar values of $\mathrm{M}(\mathrm{M}=2,4,6)$ with fixed $\mathrm{m}=0.5, \mathrm{Da}=0.1, \mathrm{k}_{1}=$ $0.1, \mathrm{dp} / \mathrm{dx}=-1, \mathrm{t}=0.4, \varnothing=\pi / 6, \mathrm{x}=0.6, \varepsilon=0.2$. It can be notice from this graph that with an increase in magnetic field parameter $\mathrm{M}$, the results in velocity profile diminished.

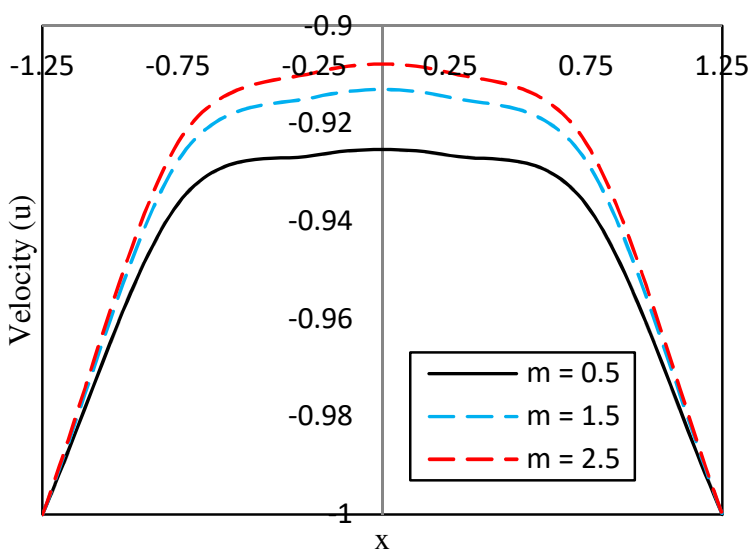

Fig. 2 Impact of $\mathrm{m}$ on axial velocity distribution with $\mathrm{Da}=0.1, \mathrm{M}=2$, $\mathrm{k}_{1}=0.1, \mathrm{dp} / \mathrm{dx}=-1, \mathrm{t}=0.4, \varnothing=\pi / 6, \mathrm{x}=0.6, \varepsilon=0.2$

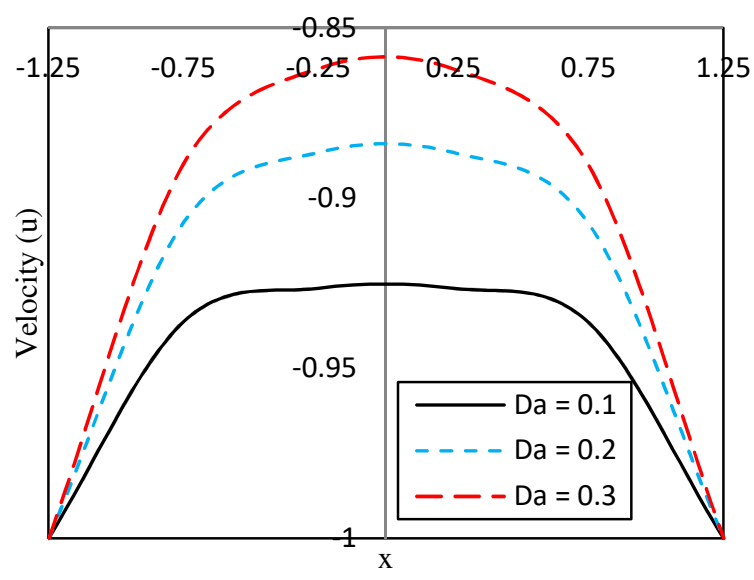

Fig. 3 Impact of Da on axial velocity distribution with $\mathrm{m}=0.5, \mathrm{M}=2$, $\mathrm{k}_{1}=0.1, \mathrm{dp} / \mathrm{dx}=-1, \mathrm{t}=0.4, \emptyset=\pi / 6, \mathrm{x}=0.6, \varepsilon=0.2$

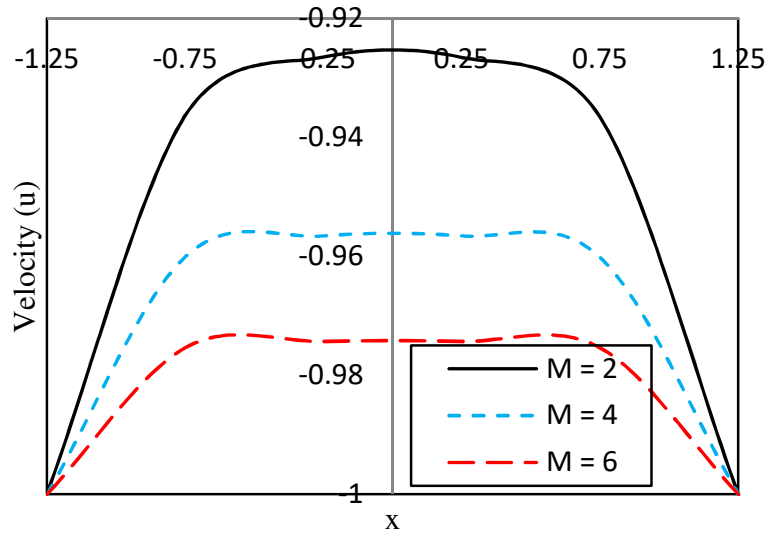

Fig. 4 Impact of $M$ on axial velocity distribution with $\mathrm{m}=0.5, \mathrm{k}_{1}=0.1$ $\mathrm{Da}=0.1, \mathrm{dp} / \mathrm{dx}=-1, \mathrm{t}=0.4, \varnothing=\pi / 6, \mathrm{x}=0.6, \varepsilon=0.2$
Fig.5 describe the influence of different hall current parameters $\mathrm{m}$ on axial pressure gradient with fixed $\mathrm{Da}=0.1, \overline{\mathrm{Q}}=0.2, \mathrm{t}=\frac{\pi}{4}, \mathrm{M}=2$, $\mathrm{k}_{1}=0.1, \mathrm{~d}=2, \emptyset=\pi / 6, \varepsilon=0.2$. We notice from this graph that the $\mathrm{dp} / \mathrm{dx}$ reduces by increase in $\mathrm{m}(\mathrm{m}=0.5,1.5,2.5)$. Influence of magnetic field parameter $\mathrm{M}$ on pressure gradient depicted in figure 6 . Indeed, the axial pressure gradient increases by increase in $\mathrm{M}(\mathrm{M}=2,4,6)$ with fixed other parameters.

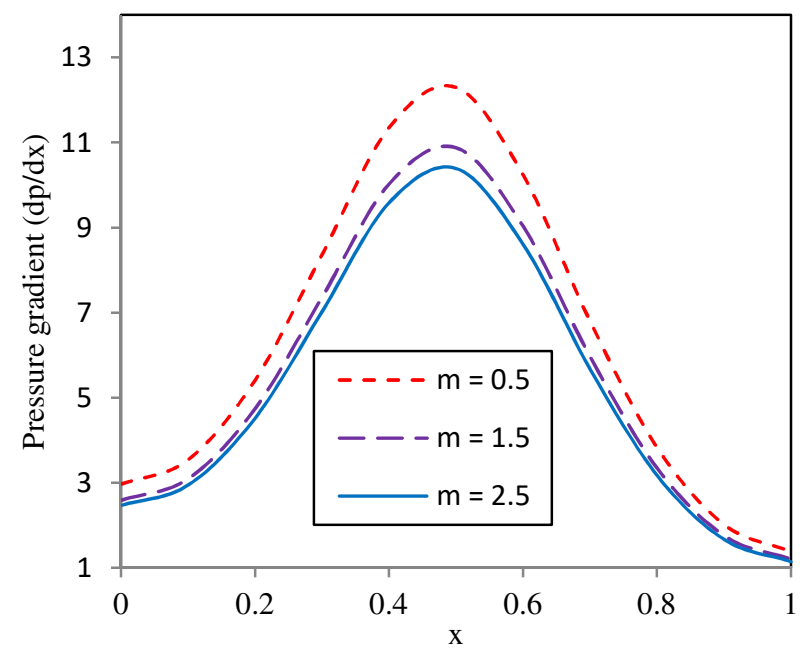

Fig. 5 Impact of $\mathrm{m}$ on $\frac{d p}{d x}$ with $\mathrm{Da}=0.1, \overline{\mathrm{Q}}=0.2, \mathrm{t}=\frac{\pi}{\mathbf{4}}, \mathrm{M}=2, \mathrm{k}_{1}=0.1$, $\mathrm{d}=2, \emptyset=\pi / 6, \varepsilon=0.2$

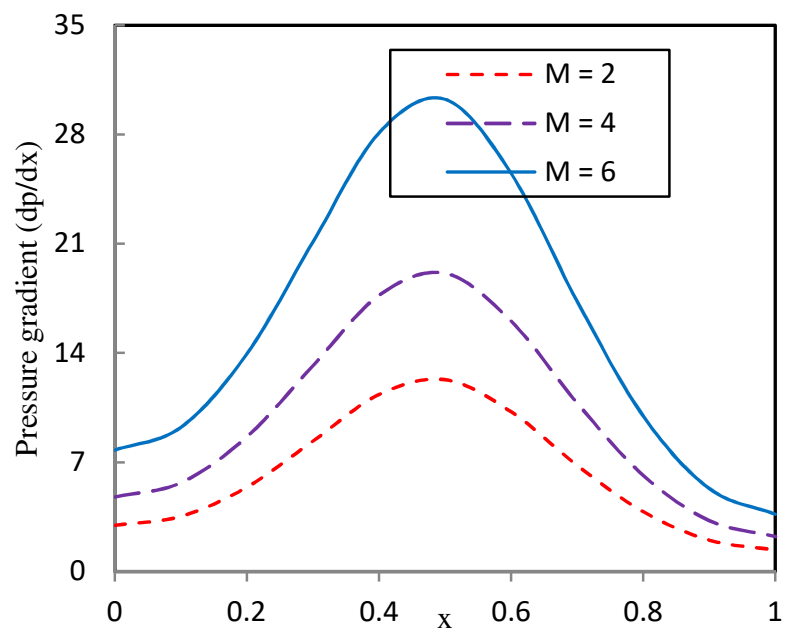

Fig. 6 Impact of $\mathrm{M}$ on $\frac{d p}{d x}$ with $\mathrm{Da}=0.1, \overline{\mathrm{Q}}=0.2, \mathrm{~m}=0.5, \mathrm{t}=\frac{\pi}{\mathbf{4}}, \mathrm{M}=2$, $\mathrm{k}_{1}=0.1, \mathrm{~d}=2, \emptyset=\pi / 6, \varepsilon=0.2$

Fig. 7 reveals the dissimilar values of Da on $\mathrm{dp} / \mathrm{dx}$ with fixed other parameters. It can be notice that the axial pressure gradient reduces by increase in Da. Figure 8 represents the flow structure of the axial pressure gradient for varied values of $\bar{Q}$ with fixed $\mathrm{M}=2, \mathrm{Da}=0.1, \quad \mathrm{~m}=0.5, \mathrm{t}$ $=\frac{\pi}{4}, \mathrm{M}=2, \mathrm{k}_{1}=0.1, \mathrm{~d}=2, \varnothing=\pi / 6, \varepsilon=0.2$. It was observed that when $\bar{Q}$ increased, the result in axial pressure gradient reduces. An important result presented in figure 9 . We observe form this graph that the $\mathrm{dp} / \mathrm{dx}$ increases in the region $x \in[0,0.5]$ and the slowly reduces in the other region $x \in[0.5,1]$ by increase in $\varphi$. 


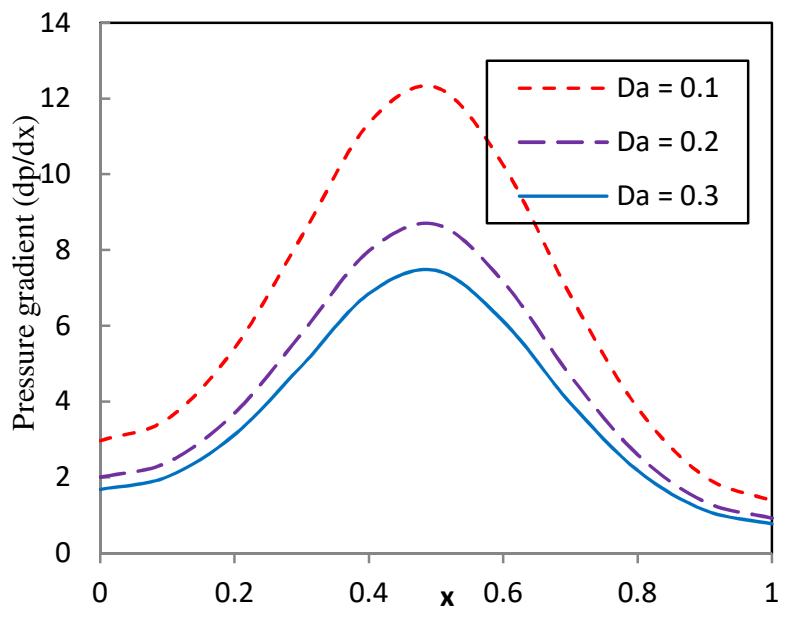

Fig. 7 Impact of Da on $\frac{d p}{d x}$ with $M=2, \bar{Q}=0.2, m=0.5, t=\frac{\pi}{4}, M=2$, $\mathrm{k}_{1}=0.1, \mathrm{~d}=2, \emptyset=\pi / 6, \varepsilon=0.2$

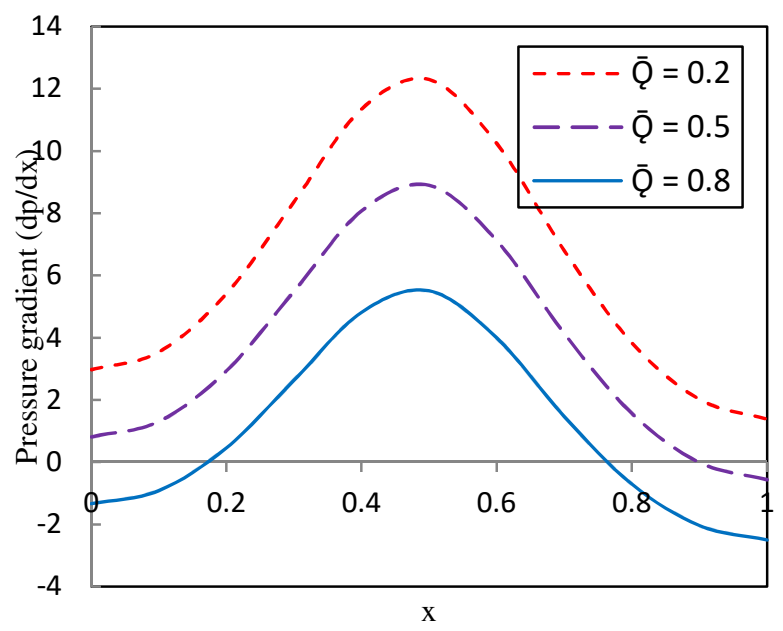

Fig. 8 Impact of $\bar{Q}$ on $\frac{d p}{d x}$ with $\mathrm{M}=2, \mathrm{Da}=0.1, \mathrm{~m}=0.5, \mathrm{t}=\frac{\pi}{4}, \mathrm{M}=2$, $\mathrm{k}_{1}=0.1, \mathrm{~d}=2, \emptyset=\pi / 6, \varepsilon=0.2$

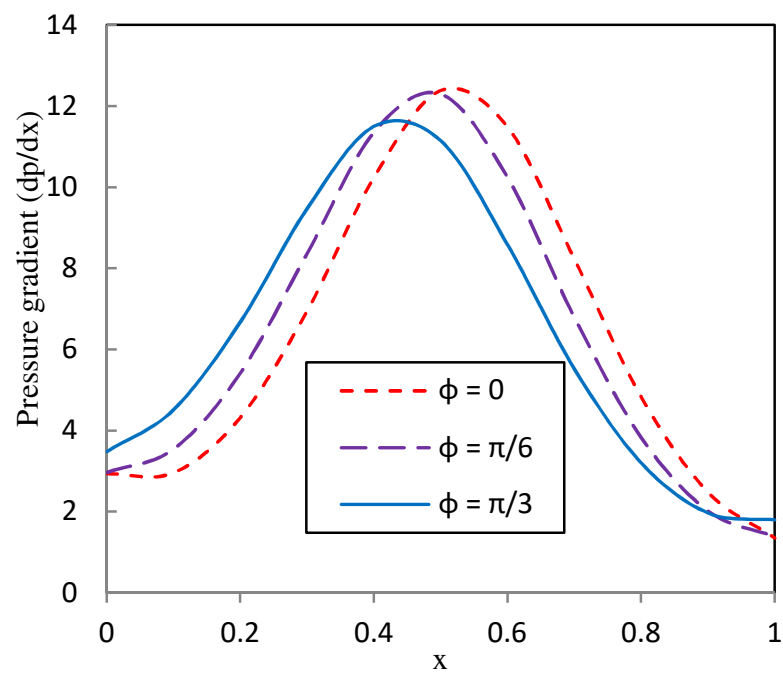

Fig. 9 Impact of $\emptyset$ on $\frac{d p}{d x}$ with $\mathrm{M}=2, \mathrm{Da}=0.1, \mathrm{~m}=0.5, \mathrm{t}=\frac{\pi}{4}, \mathrm{M}=2$, $\mathrm{k}_{1}=0.1, \mathrm{~d}=2, \overline{\mathrm{Q}}=0.2, \varepsilon=0.2$
Fig.10 presents the various values of $\mathrm{m}(\mathrm{m}=0.5,1.5,2.5)$ on temperature profile $(\theta)$. It has been inferred that the temperature distribution diminished by increase in $\mathrm{m}$ with fixed $\mathrm{Da}=0.3, \mathrm{~N}=0.4, \mathrm{Pr}$ $=1.5, \mathrm{M}=2, \beta=0.5, \mathrm{Br}=0.3, \phi=\pi / 6, \mathrm{k}_{1}=0.1, \mathrm{p}=-1, \mathrm{t}=0.4, \quad \mathrm{x}=$ $0.6, \varepsilon=0.2$. Influence of $\mathrm{M}$ on temperature distribution $(\theta)$ is displayed in fig. 11 with fixed other parameters. We perceive from this graph that the increase in $\mathrm{M}$, the results in the flow field rises. Fig.12 shows to examine the effect of $\mathrm{Da}$ on temperature distribution with fixed $\mathrm{M}=2$, $\mathrm{N}=0.4, \operatorname{Pr}=1.5, \mathrm{~m}=0.5, \beta=0.5, \mathrm{Br}=0.3, \phi=\pi / 6, \quad \mathrm{k}_{1}=0.1, \mathrm{p}=-1, \mathrm{t}$ $=0.4, \mathrm{x}=0.6, \varepsilon=0.2$. This graph indicates that the results in the temperature profile reduce by increase in Da.

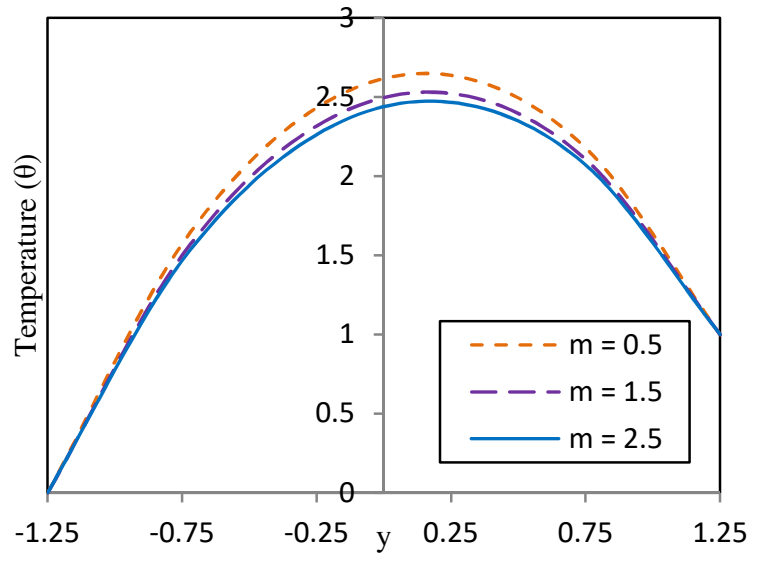

Fig. 10 Impact of $\mathrm{m}$ on $\theta$ with $\mathrm{Da}=0.3, \mathrm{~N}=0.4, \mathrm{Pr}=1.5, \mathrm{M}=2$ $\beta=0.5, \mathrm{Br}=0.3, \phi=\pi / 6, \mathrm{k}_{1}=0.1, \mathrm{p}=-1, \mathrm{t}=0.4, \mathrm{x}=0.6, \varepsilon=0.2$

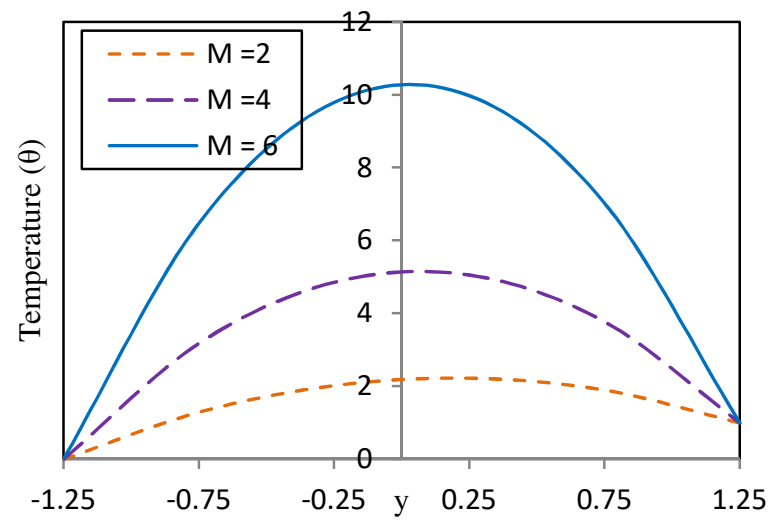

Fig. 11 Impact of $\mathrm{M}$ on $\theta$ with $\mathrm{Da}=0.1, \mathrm{~N}=0.4, \mathrm{Pr}=1.5, \mathrm{~m}=0.5$, $\beta=0.5, \mathrm{Br}=0.3, \phi=\pi / 6, \mathrm{k}_{1}=0.1, \mathrm{p}=-1, \mathrm{t}=0.4, \mathrm{x}=0.6, \varepsilon=0.2$

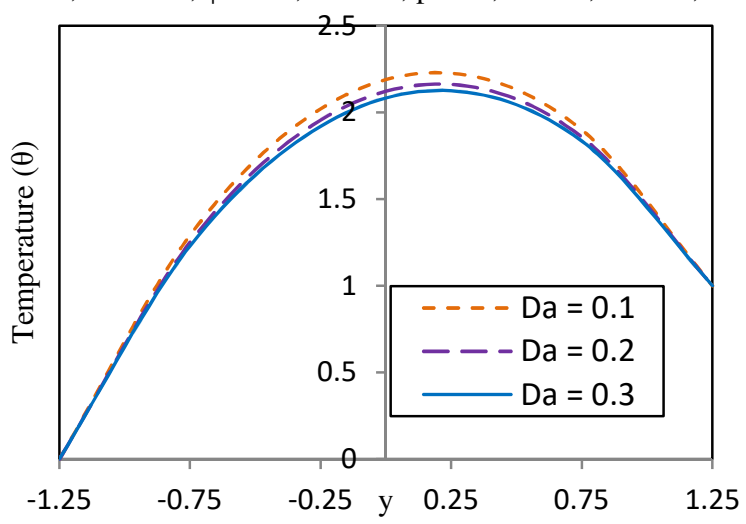

Fig. 12 Impact of $\mathrm{Da}$ on $\theta$ with $\mathrm{M}=2, \mathrm{~N}=0.4, \mathrm{Pr}=1.5, \mathrm{~m}=0.5$, $\beta=0.5, \mathrm{Br}=0.3, \phi=\pi / 6, \mathrm{k}_{1}=0.1, \mathrm{p}=-1, \mathrm{t}=0.4, \mathrm{x}=0.6, \varepsilon=0.2$ 
Fig.13 shows the temperature distribution with various values of Radiation parameter $\mathrm{N}$ with fixed other parameters. The results in temperature distribution rises by rise in N. Fig.14 depicts the temperature distribution $(\theta)$ with dissimilar values of $\operatorname{Pr}(\operatorname{Pr}=1.5,3,4.5)$ with fixed other parameters. It can be observed that from this graph that the temperature distribution enhances by increase in Pr. Effect of Brinkman number $\mathrm{Br}$ on temperature of the fluid $(\theta)$ displayed in fig.15. It clear from this figure that the temperature of the fluid rises by increase in $\mathrm{Br}$ $(\mathrm{Br}=0.3,0.5,0.7)$.

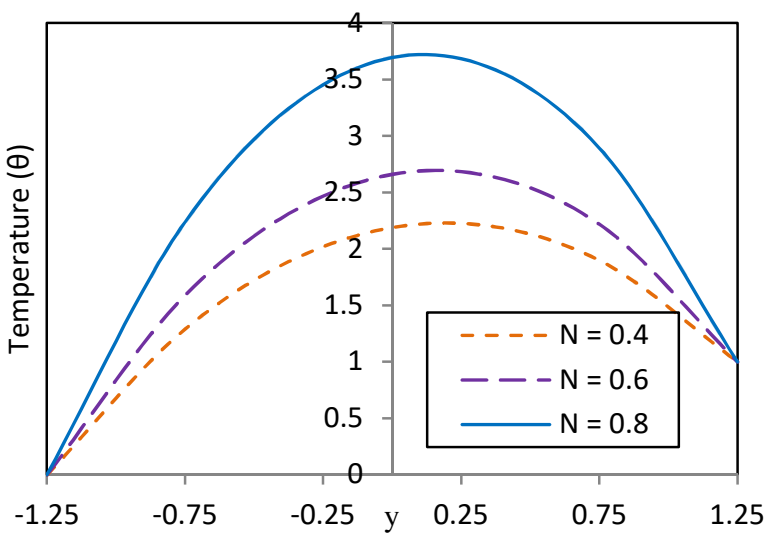

Fig. 13 Impact of $\mathrm{N}$ on $\theta$ with $\mathrm{M}=2, \mathrm{Da}=0.1, \mathrm{Pr}=1.5, \mathrm{~m}=0.5$, $\beta=0.5, \mathrm{Br}=0.3, \phi=\pi / 6, \mathrm{k}_{1}=0.1, \mathrm{p}=-1, \mathrm{t}=0.4, \mathrm{x}=0.6, \varepsilon=0.2$

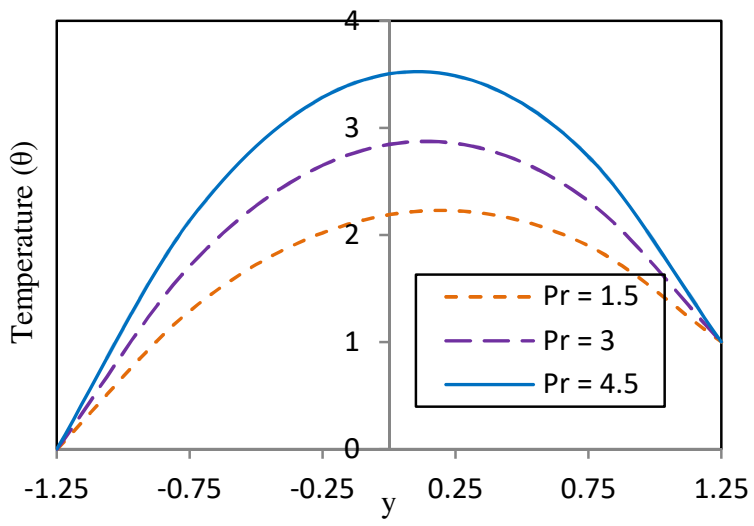

Fig. 14 Impact of $\operatorname{Pr}$ on $\theta$ with $\mathrm{M}=2, \mathrm{Da}=0.1, \mathrm{~N}=0.4, \mathrm{~m}=0.5$, $\beta=0.5, \mathrm{Br}=0.3, \phi=\pi / 6, \mathrm{k}_{1}=0.1, \mathrm{p}=-1, \mathrm{t}=0.4, \mathrm{x}=0.6, \varepsilon=0.2$

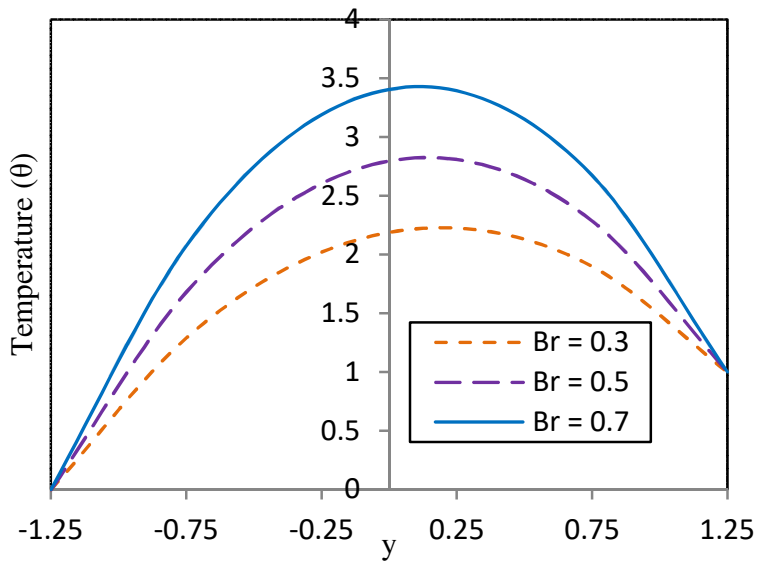

Fig. 15 Impact of $\mathrm{Br}$ on $\theta$ with $\mathrm{M}=2, \mathrm{Da}=0.1, \mathrm{~N}=0.4, \mathrm{~m}=0.5$, $\beta=0.5, \operatorname{Pr}=1.5, \phi=\pi / 6, \mathrm{k}_{1}=0.1, \mathrm{p}=-1, \mathrm{t}=0.4, \mathrm{x}=0.6, \varepsilon=0.2$
An influence of magnetic field parameter $\mathrm{M}$ on $\mathrm{Z}$ (coefficient of heat transfer) at $y=h_{1}$ wall is shown in figure 16 with fixed other parameters. It clear from this figure that with increase in $M(M=2,4,6)$, the fluid in the coefficient of heat transfer rises in $\mathrm{x} \in[0,0.04] \mathrm{U}[0.58$, $1]$ and it reduces in the other region $x \in[0.04,0.58]$. Figure 17 reveals the heat transfer coefficient with radiation parameter being fixed other parameters. It can be seen that with rise in $\mathrm{N}(\mathrm{N}=0.4,0.6,0.8)$, the results in $\mathrm{Z}$ enhances in the region $\mathrm{x} \in[0,0.04] \mathrm{U}[0.58,1]$ and the fluid in $\mathrm{Z}$ reduces in the other region $x \in[0.04,0.58]$. Effect of $\operatorname{Pr}$ on $Z$ at $y=h_{1}$ wall is shown in figure 18. It observed that with the rise in $\operatorname{Pr}$, the coefficient of heat transfer distribution $(Z)$ enhances in the region $x \in[0,0.04] \mathrm{U}$ $[0.58,1]$ whereas the results in $\mathrm{Z}$ reduces in the other region $\mathrm{x} \in[0.04$, $0.58]$.

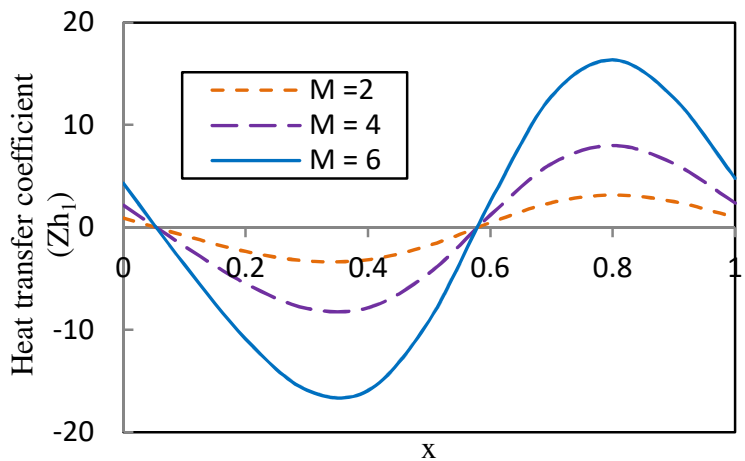

Fig. 16 Influence of $M$ on coefficient of heat transfer with $\mathrm{m}=0.5$, $\mathrm{Da}=0.1, \mathrm{~N}=0.4, \mathrm{Br}=0.3, \beta=0.5, \operatorname{Pr}=1.5, \phi=\pi / 6, \mathrm{k}_{1}=0.1$, $\mathrm{p}=-1, \mathrm{t}=0.4, \mathrm{x}=0.6, \varepsilon=0.2$

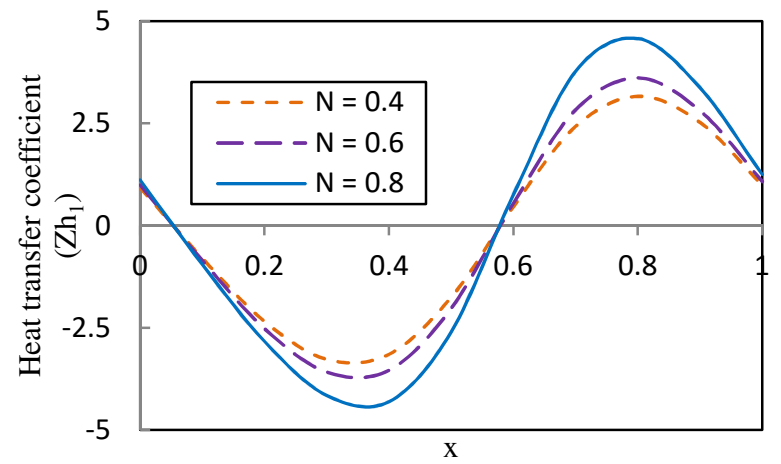

Fig. 17 Influence of $\mathrm{N}$ on coefficient of heat transfer with $\mathrm{m}=0.5$, $\mathrm{Da}=0.1, \mathrm{M}=2, \mathrm{Br}=0.3, \beta=0.5, \operatorname{Pr}=1.5, \phi=\pi / 6, \mathrm{k}_{1}=0.1, \mathrm{p}=-1$, $\mathrm{t}=0.4, \mathrm{x}=0.6, \varepsilon=0.2$

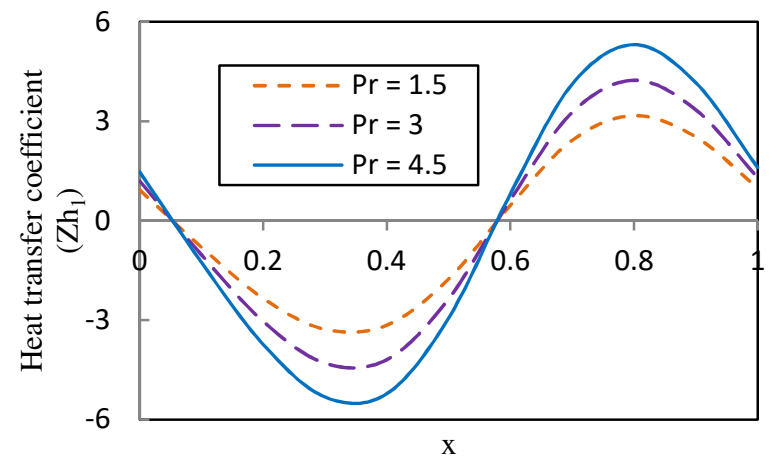

Fig. 18 Influence of $\operatorname{Pr}$ on coefficient of heat transfer with $\mathrm{m}=0.5$, $\mathrm{Da}=0.1, \mathrm{M}=2, \mathrm{Br}=0.3, \beta=0.5, \mathrm{~N}=0.4, \phi=\pi / 6, \mathrm{k}_{1}=0.1, \mathrm{p}=-1$, $\mathrm{t}=0.4, \mathrm{x}=0.6, \varepsilon=0.2$ 
Figure 19 presents the heat transfer coefficient with $\mathrm{Br}$. It was evident that from the graph that with rise $\mathrm{Br}$, the results in $\mathrm{Z}$ enhances in the region $x \in[0,0.04] U[0.58,1]$ whereas the results in $Z$ slowly reduces in the other region $x \in[0.04,0.58]$. An impact of hall current parameter $\mathrm{m}$ on $\mathrm{Z}$ (coefficient of heat transfer) at $\mathrm{y}=\mathrm{h}_{1}$ wall is shown in figure 20 with fixed other parameters. It is interesting to note that the result in coefficient of heat transfer coefficient distribution $(Z)$ reduces in the region $\mathrm{x} \in[0,0.04] \mathrm{U}[0.58,1]$ and the results in the other region $\mathrm{x} \in[0.04,0.58]$ enhances with increase in $\mathrm{m}$.

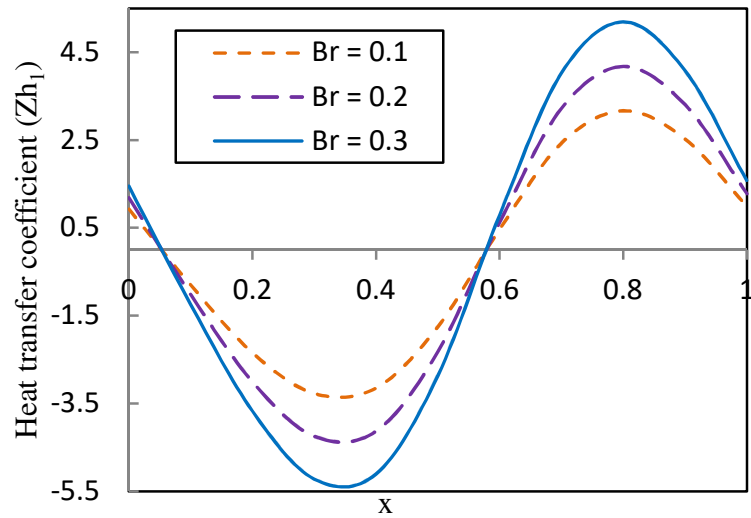

Fig. 19 Influence of $\mathrm{Br}$ on coefficient of heat transfer with $\mathrm{m}=0.5$, $\mathrm{Da}=0.1, \mathrm{M}=2, \operatorname{Pr}=1.5, \beta=0.5, \mathrm{~N}=0.4, \phi=\pi / 6, \mathrm{k}_{1}=0.1, \mathrm{p}=-1$, $\mathrm{t}=0.4, \mathrm{x}=0.6, \varepsilon=0.2$

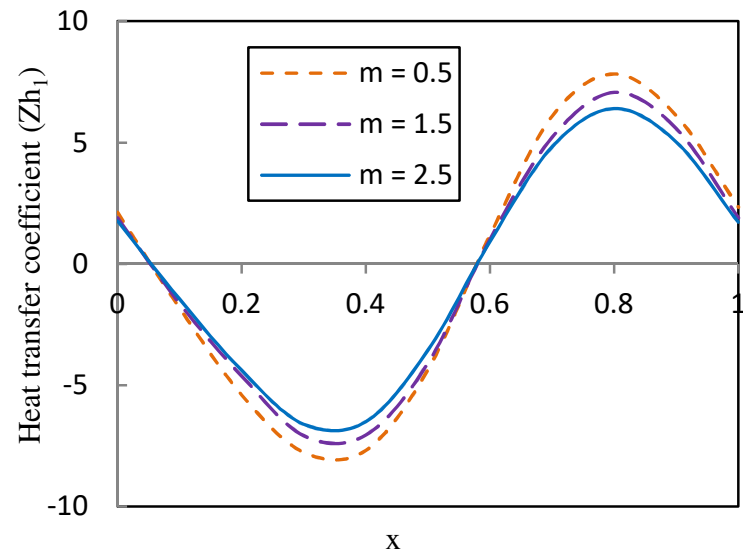

Fig. 20 Influence of $m$ on coefficient of heat transfer with $\mathrm{M}=4$, $\mathrm{Da}=0.3, \mathrm{~N}=0.4, \mathrm{Br}=0.3, \beta=0.5, \operatorname{Pr}=1.5, \phi=\pi / 6, \mathrm{k}_{1}=0.1$,

$$
\mathrm{p}=-1, \mathrm{t}=0.4, \mathrm{x}=0.6, \varepsilon=0.2
$$

\section{CONCLUSIONS}

Hall currents and joule heating on hemodynamic peristaltic flow with porous medium through a vertical tapered asymmetric channel under the influence of radiation-Blood flow analysis model have been studied through this paper. The mathematical problem is solved analytically by using long wavelength and low Reynolds number assumptions. The significant findings of this paper are summarized below.

1. The axial velocity distribution rises with rise in hall current parameter $(\mathrm{m})$ and porous parameter (Da) and the axial velocity distribution reduces when magnetic field parameter (M) increased.

2. When rise in hall current parameter $(\mathrm{m})$, porous parameter $(\mathrm{Da})$ and volumetric flow rate $(\overline{\mathbf{Q}})$, the results in axial pressure gradient diminished.
3. The temperature of the fluid increases when Magnetic field parameter $(\mathrm{M})$, Radiation parameter $(\mathrm{N})$, Prandtl number $(\mathrm{Pr})$ and Brinkman number $(\mathrm{Br})$ increased whereas the results in temperature of the fluid reduces when increase in hall current parameter $(\mathrm{m})$ and porous parameter $(\mathrm{Da})$ increased.

4. Heat transfer coefficient rises in $\mathrm{x} \in[0,0.04] \mathrm{U}[0.58,1]$ and it reduces in the other region $x \in[0.04,0.58]$ with increase in $\mathrm{M}, \mathrm{N}, \mathrm{Pr}$ and $\mathrm{Br}$.

5. Heat transfer coefficient distribution $(\mathrm{Z})$ reduces in the region $\mathrm{x} \in[0,0.04] \mathrm{U}[0.58,1]$ and the results in the other region $\mathrm{x} \in$ $[0.04,0.58]$ enhances with increase in hall currents parameter $\mathrm{m}$.

\section{REFERENCES}

Abd El-Maboud, Y., Mekheimer, K. S., 2010, "Non-linear Peristaltic Transport of a Second-order Fluid through a Porous Medium," Applied Mathematical Modelling, 35 (6), 2695-2710. https://doi.org/10.1016/j.apm.2010.11.031

Abzal, S.K., Vijaya Kumar Varma, S., Ravikumar, S., 2016, "Influence of Heat Transfer on Magnetohydrodynamic Peristaltic Blood Flow with Porous Medium through a Coaxial Vertical Asymmetric Tapered Channel - An Analysis of Blood Flow Study," International Journal of Engineering Sciences \& Research Technology, 5(4), 896-915. http://dx.doi.org/10.5281/zenodo.50433

Agarwal, H. L., Anwaruddin. B., 1984, "Peristaltic Flow of Blood a Branch," Ranchi University Mathematical Journal, 15,111-118.

Akbar, N. S., Nadeem, S., 2010, "Influence of Heat Transfer on Peristaltic Transport of a Johnson-Segalman Fluid in an Inclined Asymmetric Channel," Communications in Nonlinear Science and Numerical Simulation, 15(10), 2860-2877. https://doi.org/10.1016/j.cnsns.2009.10.030

Ali, N., Sajid, M., Javed, T., Abbas, Z., 2010, "Heat transfer Analysis of Peristaltic Flow in a Curved Channel," International Journal of Heat and Mass Transfer, 53 (15-16), 3319-3325. http://dx.doi.org/10.1016/j.ijheatmasstransfer.2010.02.036

Asghar, S., Mohyuddin, M. R., Hayat, T., 2005, "Effects of Hall Current and Heat Transfer on Flow due to a Pull of Eccentric Rotating Disks," International Journal of Heat and Mass Transfer, 48(3-4), 599-607. https://doi.org/10.1016/j.ijheatmasstransfer.2004.08.023

Attia, H. A., 2004, "Unsteady Hartmann Flow with Heat Transfer of a Viscoelastic Fluid considering the Hall Effect," Canadian Journal of Physics, 82 (2), 127-139. https://doi.org/10.1139/p03-117

Cogley, A. C. L., Vinvent, W. G., Giles, E. S.,1968, "Differential Approximation for Radiative Heat Transfer in Non-linear EquationsGrey Gas near Equilibrium," American Institute of Aeronautics and Astronautics, 6, 551-553.

Eldabe, N., Ghaly, A., Sallam, S., Elagamy, K., Younis, Y., 2015, "Hall Effect on Peristaltic Flow of Third Order Fluid in a Porous Medium with Heat and Mass Transfer," Journal of Applied Mathematics and Physics, 3(9), 1138-1150.

http://dx.doi.org/10.4236/jamp.2015.39141 
El Naby, A. E. H. A., El Misery, A. E. M., Abd El Kareem, M. F.2006, "Effects of a Magnetic Field on Trapping through Peristaltic Motion for Generalized Newtonian Fluid in Channel," Physica A, 367, 79-92.

https://doi.org/10.1016/j.physa.2005.10.045

Fung, Y. C., Yih, C. S., 1968, "Peristaltic Transport," Journal of Applied Mechanics, 35(4), 669-675.

http://dx.doi.org/10.1115/1.3601290

Gharsseldien, Z. M., Mekheimer, K. S., Awad, A.S., 2010, "The Influence of Slippage on Trapping and Reflux limits with Peristalsis through an Asymmetric Channel," Applied Bionics and Biomechanics, 7(2), 95-108.

https://doi.org/10.1080/11762321003747099

Haroun, M. H., 2007, "Non-linear Peristaltic Transport Flow of a Fourth Grade Fluid in an Inclined Asymmetric Channel," Computational Materials Science, 39, 324-333.

https://doi.org/10.1016/j.commatsci.2006.06.012

Hayat, T., Ali, N., Asghar, S., 2007, "Hall Effects on Peristaltic Flow of a Maxwell Fluid in a Porous Medium," Physics Letters A, 363(5-6), 397403.

https://doi.org/10.1016/j.physleta.2006.10.104

Hayat, T., Hina Zahir, Anum Tanveer, Alsaedi, A., 2016, "Influences of Hall Current and Chemical Reaction in Mixed Convective Peristaltic Flow of Prandtl Fluid," Journal of Magnetism and Magnetic Materials, 407, 321-327.

https://doi.org/10.1016/j.jmmm.2016.02.020

Jaffrin, M. Y., Shapiro, A. H., 1971, "Peristaltic Pumping," Annual Review of Fluid Mechanics, 3(1), 13-36.

Jitendra Kumar Singh, Naveen Joshi, Shik Ghousia Begum, Srinivasa, C.T., 2016, "Unsteady Hydromagnetic Heat and Mass Transfer Natural Convection Flow Past an Exponentially Accelerated Vertical Plate with Hall Current and Rotation in the Presence of Thermal and Mass Diffusions," Frontiers in Heat and Mass Transfer (FHMT), 7 - 24, 1-12. http://dx.doi.org/10.5098/hmt.7.24

Kothandapani, M., Prakash, J., Pushparaj, V., 2015, “Analysis of Heat and Mass Transfer on MHD Peristaltic Flow through a Tapered Asymmetric Channel," Journal of Fluids, 2015, 1-9.

\section{http://dx.doi.org/10.1155/2015/561263}

Latham, T.W., 1966, "Fluid Motion in a Peristaltic Pump," MSc Thesis, Massachusetts Institute of Technology, Cambridge Massachusetts.

Mekheimer, K. S., 2004, "Peristaltic flow of Blood under Effect of a Magnetic Field in a Non-uniform Channels," Applied Mathematics and Computation, 153(3), 763-777.

https://doi.org/10.1016/S0096-3003(03)00672-6

Mekheimer, K. S., Husseny, S. A., Abd El-Maboud, Y., 2010, "Effects of Heat Transfer and Space Porosity on Peristaltic Flow in a Vertical Asymmetric Channel," Numerical Methods of Partial Differential Equations Journal, 26(4), 747-770.

https://doi.org/doi:10.1002/num.20451

Mishra, M., Ramachandra Rao, A., 2003, "Peristaltic Transport of a Newtonian Fluid in an Asymmetric Channel," Zeitschrift fur Angewandte Mathematik und Physik, 54 (3), 532-550. https://doi.org/10.1007/s00033-003-1070-7

Mustafa, M., Abbasbandy, S., Hina, S., Hayat, T., 2014, "Numerical Investigation on Mixed Convective Peristaltic Flow of Fourth-Grade Fluid with Dufour and Soret Effects," J Taiwan Institute of Chemical Engineers, 45, 308-316.

https://doi.org/10.1016/j.jtice.2013.07.010

Nadeem, S., Hayat, T., Akbarand, N. S., Malik, M. Y., 2009, "On the Influence of Heat Transfer in Peristalsis with Variable Viscosity," Int. J.Heat Mass Transfer, 52, 4722-4730.

https://doi.org/10.1016/j.ijheatmasstransfer.2009.04.037

Nowar, K. 2014, "Peristaltic Flow of a Nanofluid under the Effect of Hall Current and Porous Medium," Mathematical Problems in Engineering, 2014, 1-15

http://dx.doi.org/10.1155/2014/389581

Ravikumar, S., 2016, "Analysis of Heat Transfer on MHD Peristaltic Blood Flow with Porous Medium through Coaxial Vertical Tapered Asymmetric Channel with Radiation - Blood Flow Study," International Journal of Bio-Science and Bio-Technology, 8(2), 395-408. http://dx.doi.org/10.14257/ijbsbt.2016.8.2.37

Shapiro, A. H., Jaffrin, M. Y., Weinberg, S. L., 1969, "Peristaltic Pumping with Long Wavelengths at Low Reynolds Number," Journal of Fluid Mechanics, 37(4), 799-825. https://doi.org/10.1017/S0022112069000899

Shehawey, E. F. EI., Husseny, S. Z. A., 2002, "Peristaltic Transport of a Magneto-Fluid with Porous Boundaries," Applied Mathematics and Computation, 129 (2-3), 421-440. https://doi.org/10.1016/S0096-3003(01)00054-6

Shehzad, S. A., Abbasi, F. M., Hayat, T., Alsaadi, F., 2014, "MHD Mixed Convective Peristaltic Motion of Nanofluid with Joule Heating and Thermophoresis Effects," PLoS ONE, 9(11), e111417.

https://doi.org/10.1371/journal.pone.0111417

Siddiqui, A. M., Hayat, T., Khan, M., 2004, "Magnetic fluid model induced by peristaltic waves," Journal of the Physical Society of Japan, 73 (8), 2142-2147.

http://dx.doi.org/10.1143/JPSJ.73.2142

Srinivas, S., Muthuraj, R., 2011, "Effects of Chemical Reaction and Space Porosity on MHD Mixed Convective Flow in a Vertical Asymmetric Channel with Peristalsis," Mathematical and Computer Modelling, 54(5-6), 2011, 1213-1227.

https://doi.org/10.1016/j.mcm.2011.03.032

Srivastava, L. M., Srivastava, V. P., 1984, "Peristaltic Transport of Blood: Casson Model. II," Journal of Biomechanics, 17(11), 821-829. http://dx.doi.org/10.1016/0021-9290(84)90140-4

Tasawar Hayat, Hina Zahir, Ahmed Alsaedi, Bashir Ahmad, 2017, "Hall Current and Joule heating Effects on Peristaltic Flow of Viscous Fluid in a Rotating Channel with Convective Boundary Conditions," Results in Physics, 7, 2831-2836.

https://doi.org/10.1016/j.rinp.2017.07.069

Vajravelu, K., Radhakrishnamacharya, G., Radhakrishnamurty, V., 2007, "Peristaltic Flow and Heat Transfer in a Vertical Porous Annulus with Long Wave Approximation," Int. J. Nonlinear Mech., 42, 754-759. https://doi.org/10.1016/j.ijnonlinmec.2007.02.014 
Vajravelu, S., Sreenadh, S., Lakshminarayana, P., 2011, “The Influence of Heat Transfer on Peristaltic Transport of a Jeffrey Fluid in a Vertical Porous Stratum," Communications in Nonlinear Science and Numerical Simulation, 16(8), 3107-3125.

https://doi.org/10.1016/j.cnsns.2010.11.001
Veeresh, C., Varma, S. V. K., Vijaya Kumar, A.G., Umamaheswar, M., M. C. Raju, M.C., 2017, "Joule Heating and Thermal Diffusion Effects on MHD Radiative and Convective Casson Fluid Flow Past an Oscillating Semi-infinite Vertical Porous Plate," Frontiers in Heat and Mass Transfer (FHMT), 8, 1-8.

http://dx.doi.org/10.5098/hmt.8.1 